\title{
29-kDa FN-f inhibited autophagy through modulating localization of HMGB1 in human articular chondrocytes
}

\author{
Hyun Sook Hwang ${ }^{1,2}$, Min Ha Choi ${ }^{1,2}$ E Hyun Ah Kim ${ }^{1,2, *}$ \\ ${ }^{1}$ Division of Rheumatology, Department of Internal Medicine, Hallym University Sacred Heart Hospital, Anyang $14068,{ }^{2}$ Institute for \\ Skeletal Aging, Hallym University, Chunchon 24251, Korea
}

Fibronectin fragments found in the synovial fluid of patients with osteoarthritis (OA) induce the catabolic responses in cartilage. Nuclear high-mobility group protein Box 1 (HMGB1), a damage-associated molecular pattern, is responsible for the regulation of signaling pathways related to cell death and survival in response to various stimuli. In this study, we investigated whether changes induced by 29-kDa aminoterminal fibronectin fragment (29-kDa FN-f) in HMGB1 expression influences the pathogenesis of OA via an HMGB1modulated autophagy signaling pathway. Human articular chondrocytes were enzymatically isolated from articular cartilage. The level of mRNA was measured by quantitative real-time PCR. The expression of proteins was examined by westem blot analysis, immnunofluorescence assay, and enzyme-linked immunosorbent assay. Interaction of proteins was evaluated by immunoprecipitation. The HMGB1 level was significantly lower in human OA cartilage than in normal cartilage. Although 29-kDa FN-f significantly reduced the HMGB1 expression at the mRNA and protein levels $6 \mathrm{~h}$ after treatment, the cytoplasmic level of HMGB1 was increased in chondrocytes treated with 29-kDa FN-f, which significantly inhibited the interaction of HMGB1 with Beclin-1, increased the interaction of Bcl-2 with Beclin-1, and decreased the levels of Beclin-1 and phosphorylated Bcl-2. In addition, the level of microtubule-associated protein 1 light chain 3-II, an autophagy marker, was down-regulated in chondrocytes treated with 29-kDa FN-f, whereas the effect was antagonized by mTOR knockdown. Furthermore, prolonged treatment with 29-kDa FN-f significantly increased the release of HMGB1 into the culture medium. These results demonstrated that 29-kDa FN-f inhibits chondrocyte autophagy by modulating the HMGB1 signaling pathway. [BMB Reports 2018; 51(10): 508-513]

*Corresponding author. Tel: +82-31-380-1826; Fax: +82-31-3818812; E-mail: kimha@hallym.ac.kr

https://doi.org/10.5483/BMBRep.2018.51.10.058

Received 19 March 2018, Revised 2 April 2018, Accepted 4 May 2018

Keywords: Autophagy, Chondrocyte, Fibronectin fragment, HMGB1, Osteoarthritis

\section{INTRODUCTION}

Osteoarthritis $(\mathrm{OA})$, the most common type of degenerative joint disease, causes joint pain and disability (1). Several risk factors, including aging, gender, genetic predisposition, and obesity, are involved in the pathogenesis of OA. Degeneration of cartilage extracellular matrix (ECM), the major components of which are proteoglycans and collagens, is a key pathologic finding in OA. A variety of cytokines, such as interleukin (IL)-1 and tumor necrosis factor- $\alpha$ (TNF- $\alpha$ ), lead to imbalance of cartilage homeostasis, subsequently degrading ECM by increasing inflammatory mediators, such as nitric oxide (NO) and prostaglandin E2, and catabolic enzymes, such as matrix metalloproteinases (MMPs) and a disintegrin and metalloproteinase with thrombospondin motifs (2).

Autophagy is an important process for the maintenance of cellular homeostasis in response to various types of stress. Several studies demonstrated that the defect of autophagy leads to diverse diseases, including neurodegenerative diseases and OA (3). There is accumulating evidence that disturbance of autophagy is related to chondrocyte death and survival. Because chondrocytes, the single type of cells embedded in articular cartilage, are responsible for ECM homeostasis, such as synthesis and turnover, regulation of chondrocyte survival is crucial in the pathogenesis of OA (4). Autophagic markers in the articular cartilage of normal humans, sham, and young mice were higher than those in human OA, surgically induced OA and aging mice, respectively, indicating that autophagy may serve as a protective mechanism $(5,6)$. Autophagy was suppressed in the superficial zone of articular cartilage in response to mechanical injury (7).

Fibronectin ( $\mathrm{FN}$ ) is an ECM glycoprotein present in cartilage and synovial fluid (8). Fibronectin fragments (FN-fs) of 29-, 45-, $120-$, and $200-\mathrm{kDa}$ derived from $\mathrm{FN}$ are found in OA cartilage and synovial fluid, and they stimulate the production of various inflammatory cytokines, such as TNF- $\alpha$ and IL-1 $\beta$ (9, 10). In particular, 29-kDa fibronectin fragment (29-kDa FN-f), the most potent molecule, is associated with proteoglycan breakdown by up-regulating NO, catabolic cytokines, and MMP family proteins in articular chondrocytes $(11,12)$. Previously, we reported that $29-\mathrm{kDa} \mathrm{FN}-\mathrm{f}$ stimulates expression of catabolic factors through the toll-like receptor 2 (TLR-2)- 
dependent signaling pathway (13). However, it is unknown whether 29-kDa FN-f regulates the autophagy pathway in chondrocytes.

High-mobility group box 1 (HMGB1), a non-histone DNA-binding protein in the nuclei, regulates transcription and DNA repair in the nucleus (14). Previous studies demonstrated that HMGB1 is a potent regulator of autophagy, and translocation of nuclear HMGB1 into cytoplasm by cellular stress induces autophagy (14). Cytoplasmic HMGB1 by binding to Beclin-1 leads to autophagy, whereas a complex of Beclin-1 and Bcl-2 suppresses it (15). In addition, HMGB1 is released into extracellular spaces in response to a variety of stimuli and works as a damage-associated molecular pattern (DAMP). For example, HMGB1 induces cellular senescence after release in a p53-dependent manner $(16,17)$. A previous report showed that HMGB1 increases pro-inflammatory responses in chondrocytes in the presence of $29-\mathrm{kDa} \mathrm{FN}-\mathrm{f}$ more than does treatment with HMGB1 alone (18).

In this study, we investigated whether 29-kDa FN-f regulates HMGB1 expression, its extracellular release, and HMGB1mediated autophagy in human articular chondrocytes.

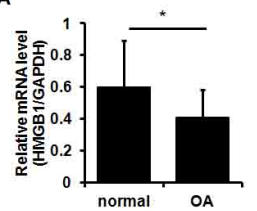

B

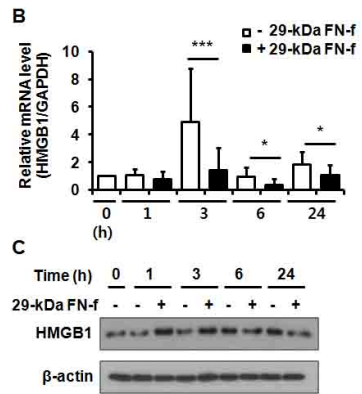

Fig. 1. High-mobility group box 1 (HMGB1) expression was modulated by 29-kDa FN-f. (A) Relative expression of HMGB1 in human normal and osteoarthritis (OA) cartilage. Relative expression of HMGB1 in normal and OA cartilage was measured using SYBR Green-based real-time polymerase chain reaction (PCR). Glyceraldehyde 3-phosphate dehydrogenase (GAPDH) was used as the endogenous control. ${ }^{*} P<0.05$ vs. normal cartilage. Data are presented as the mean \pm standard deviation (SD) of data from duplicate experiments using cartilage from different donors (normal cartilage, $n=9$ and OA cartilage, $n=20$ ). (B, C) mRNA and protein expression of HMGB1 altered by 29-kDa FN-f. Chondrocytes were stimulated with 29-kDa FN-f (300 nM) for 1, 3,6 , and 24 h. mRNA and protein levels of HMGB1 were measured using (B) qRT-PCR and (C) western blot analysis, respectively. ${ }^{*} \mathrm{P}<0.05$ and $* * * \mathrm{P}<0.005$ vs. untreated cells at each time point. Data are presented as the mean \pm SD of data from duplicate data using chondrocytes from three different donors.

\section{RESULTS}

\section{9-kDa FN-f altered HMGB1 expression in human articular chondrocytes}

To reveal the effect of HMGB1 on the pathogenesis of OA, the level of HMGB1 in the normal and OA cartilages was examined. HMGB1 expression in OA cartilage was significantly reduced much more than in normal cartilage (Fig. 1A). We next examined the effect of 29-kDa FN-f on HMGB1 expression in primary human chondrocytes. HMGB1 expression was maximally induced at $3 \mathrm{~h}$ in the control culture, whereas 29-kDa FN-f suppressed it at 3, 6, and $24 \mathrm{~h}$ (Fig. 1B). On the other hand, the level of HMGB1 protein increased at the early phase ( 1 and $3 \mathrm{~h}$ incubation) and decreased at the late phase (6 and $24 \mathrm{~h}$ incubation) in chondrocytes treated with 29-kDa FN-f (Fig. 1C).

29-kDa FN-f induced cytoplasmic translocation of HMGB1 and subsequently released HMGB1 into extracellular space We examined whether 29-kDa FN-f induces the extracellular secretion of HMGB1. Western blot analysis showed that the
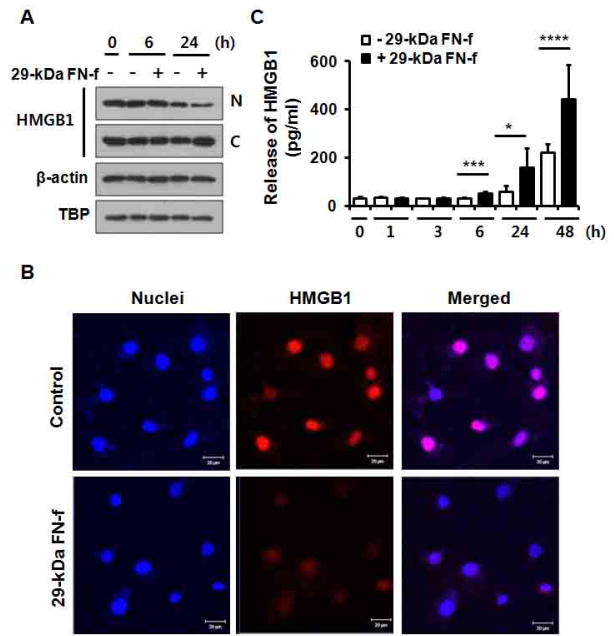

Fig. 2. Translocation of nuclear HMGB1 was increased by 29-kDa FN-f. (A, B) 29-kDa FN-f induced the cytoplasmic localization of HMGB1. Cytoplasmic and nuclear fractions were isolated from chondrocytes treated with 29-kDa FN-f for 6 and $24 \mathrm{~h}$. TATAbinding protein (TBP) and $\beta$-actin were used as loading controls for nuclear and cytoplasmic fraction, respectively. (B) Localization of HMGB1 was evaluated in primary chondrocytes in the presence or absence of $29-\mathrm{kDa} \mathrm{FN}-\mathrm{f}$ using fluorescence microscopy. Nuclei were stained with DAPI. Scale bars $=20 \mu \mathrm{m}$. (C) Release of HMGB1 into the extracellular milieu was increased by prolonged treatment with 29-kDa FN-f. Culture medium was collected from primary chondrocytes treated with 29-kDa FN-f. The levels of HMGB1 released into the medium were measured by enzyme-linked immunosorbent assay (ELISA). Data are expressed as the mean \pm SD of duplicate data from more than three independent experiments. ns, not significant, ${ }^{*} \mathrm{P}<0.05$, $* * * P<0.005$, and $* * * * P<0.001$ vs. untreated cells. 
level of HMGB1 in the cytoplasmic fractions was increased, whereas that of the nuclear fraction decreased at $24 \mathrm{~h}$ after stimulation with 29-kDa FN-f (Fig. 2A). Fluorescence microscopy revealed the down-regulation of nuclear HMGB1 in 29-kDa FN-f-stimulated cells (Fig. 2B). Enzyme-linked immunosorbent assay (ELISA) data showed that HMGB1 was released into the culture media throughout the 48-h culture period, and 29-kDa $\mathrm{FN}-\mathrm{f}$ led to its increase (Fig. 2C). The results demonstrate that 29-kDa FN-f stimulates the translocation of nuclear HMGB1 into the cytoplasm and subsequent release of HMGB1 into the extracellular space.

29-kDa FN-f inhibited autophagy through a mammalian target of rapamycin (mTOR)/HMGB1-dependent signaling pathway

We investigated whether 29-kDa FN-f inhibits the autophagy signaling pathway. We measured the level of microtubuleassociated protein 1 light-chain 3-II (LC3-II), an autophagy marker, using immmunoblot and fluorescence microscopy

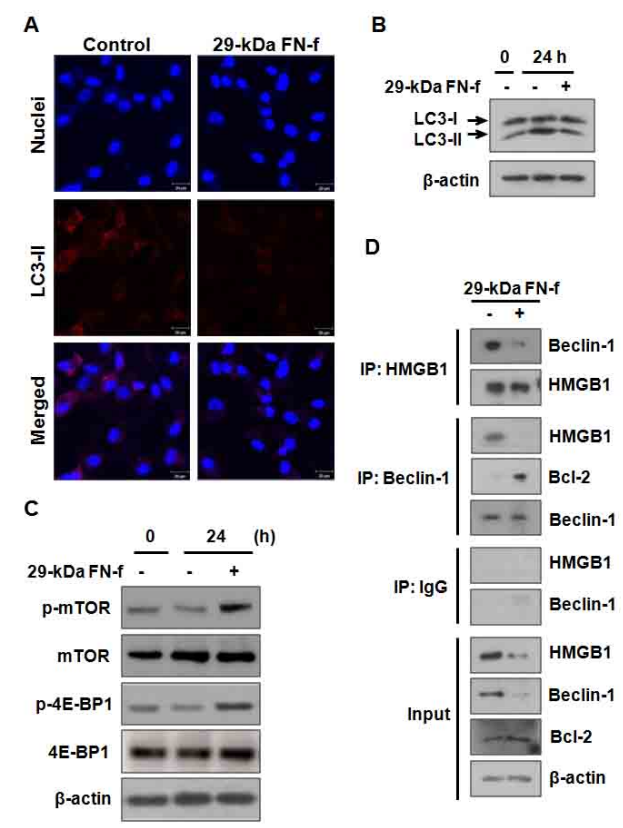

Fig. 3. Autophagy was inhibited by $29-k \mathrm{Da}$ FN-f. (A, B) LC3-II level was decreased by 29-kDa FN-f. LC3-Il level was measured by (A) immunofluorescence microscopy and (B) western blot analysis. Nuclei were stained with DAPI. (C) Activation of mTOR and 4E-BP1 by 29-kDa FN-f. Phosphorylation of mTOR and $4 \mathrm{E}-\mathrm{BP} 1$ in chondrocytes treated with 29-kDa FN-f was measured by western blot analysis. (D) The formation of HMGB1/Beclin-1 complex was diminished by 29-kDa $\mathrm{FN}-\mathrm{f}$. Interaction with HMGB1 and Beclin-1 in chondrocytes untreated and treated with 29-kDa FN-f was assessed by using an immunoprecipitation (IP) assay. After IP of cell lysates with an antibody against HMGB1, Beclin-1 or IgG, protein samples were subjected to western blot assay. IP with IgG served as a negative control. analysis. The level of LC3-II was significantly decreased by 24 h treatment with 29-kDa FN-f (Fig. 3A and B), indicating that 29-kDa FN-f significantly suppresses autophagy. Western blot analysis demonstrated that 29-kDa FN-f elevated phosphorylation of mTOR, an inhibitor of autophagy (Fig. 3C). In addition, the level of phospho-elF4E-binding protein 1 (4E-BP1), the substrate of mTOR, was increased in the presence of 29-kDa FN-f (Fig. 3C).

HMGB1 leads to autophagy when it forms a complex with Beclin-1. On the other hand, the association of Beclin-1 and Bcl-2 inhibits autophagy $(15,19)$. We examined whether $29-\mathrm{kDa}$ FN-f affects the formation of the Beclin-1/Bcl-2 and Beclin-1/HMGB1 complex. After $24 \mathrm{~h}$ exposure to 29-kDa $\mathrm{FN}-\mathrm{f}$, cell lysates were immunoprecipitated with antibodies against Beclin-1 or HMGB1. Immunoprecipitation (IP) data showed that 29-kDa FN-f elevated the formation of Bcl-2/Beclin-1 complex, which inhibits autophagy pathway, and suppressed the formation of the HMGB1/Beclin-1 complex, which induces the HMGB1-dependent autophagy pathway (Fig. 3D). Therefore, these results demonstrated that 29-kDa FN-f not only activated the mTOR signaling pathway, but also induced the interaction of Bcl-2/Beclin-1 rather than the HMGB1/Beclin-1 complex, subsequently inhibiting the mTOR/HMGB1-dependent autophagy pathway.

mTOR knockdown antagonized the 29-kDa FN-f-inhibited autophagy signaling pathway

To find out whether 29-kDa FN-f regulates autophagy and

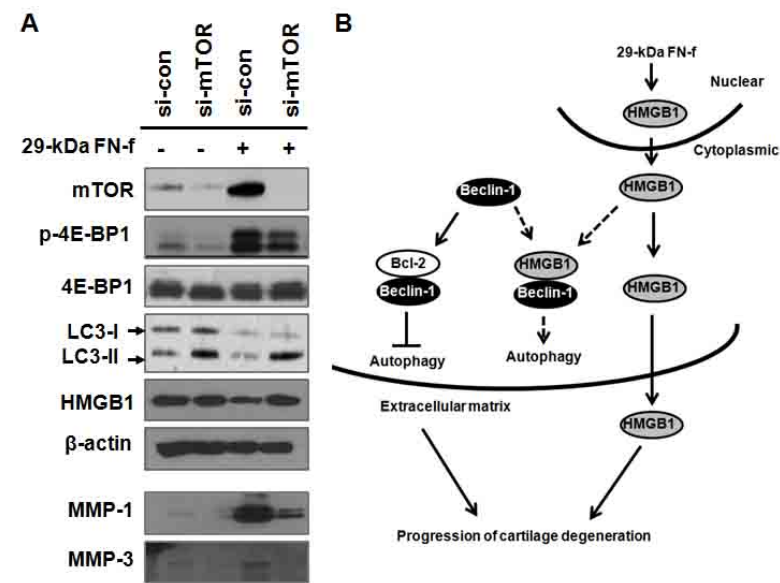

Fig. 4. mTOR silencing restored the 29-kDa FN-f-suppressed LC3-II level and HMGB1 in primary chondrocytes. (A) Chondrocytes were transfected with control siRNA (si-con) and small interfering mTOR RNA (si-mTOR); $48 \mathrm{~h}$ later chondrocytes were stimulated with 29-kDa FN-f for $24 \mathrm{~h}$. The protein levels were measured using western blot analysis. $\beta$-actin served as loading controls. (B) Schematic diagram of mechanism leading to $29-\mathrm{kDa}$ FN-f-suppressed HMGB1-dependent autophagy pathway in primary chondrocytes. 
catabolic responses through the mTOR signaling pathway, chondrocytes were transfected using si-mTOR or control siRNA, followed by stimulation with 29-kDa FN-f (300 nM) for $24 \mathrm{~h}$. Western blot analysis revealed that mTOR silencing decreased phosphorylation of 4E-BP1 and subsequently increased the level of LC3-II, an autophagy marker, more than did si-control-transfected chondrocytes in the control culture (Fig. 4A). Although the phosphorylation of 4E-BP1 increased and the expression of LC3-II and HMGB1 decreased in chondrocytes treated with 29-kDa FN-f, mTOR knockdown attenuated the phosphorylation of $4 \mathrm{E}-\mathrm{BP} 1$ and reversed the level of LC3-II and HMGB1 (Fig. 4A). In addition, 29-kDa FN-f strongly up-regulated MMP-1 and MMP-3, whereas mTOR knockdown significantly suppressed both MMP-1 and -3 . These results indicate that $29-\mathrm{kDa} \mathrm{FN}-\mathrm{f}$ negatively modulated autophagy as well as catabolic response via the mTOR signaling pathway.

\section{DISCUSSION}

We previously reported that $29-k D a \mathrm{FN}-f$ potently induced the expression of catabolic factors, including MMP-1, -3 , and -13 (13). In this study, we investigated whether 29-kDa FN-f regulates HMGB1 expression and cytoplasmic translocation. The modulation of HMGB1-dependent autophagy signaling in human articular chondrocytes was also investigated. Our results show that 29-kDa FN-f elevates extracellular release of HMGB1 and inhibits autophagy signaling by activating the mTOR signaling pathway as well as by suppressing the HMGB1/Beclin-1 complex (Fig. 4B).

The role of DAMPs, such as HMGB1, hyaluronan fragments, and $\mathrm{FN}$-fs in the induction of a vicious cycle of OA cartilage destruction has been postulated, because they are produced in the process of matrix degradation and in turn increase expression of inflammatory cytokines and MMPs (20). Increased levels of FN and FN-fs of 30-200 kDa are found in cartilage and synovial fluid from patients with $O A$ and $R A(10,21,22)$. In line with its role as a DAMP, 29-kDa FN-f increased the expression of MMPs via the TLR-2 signaling pathway (13). Of the HMGB protein family members, including HMGB1, 2, 3, and 4, HMGB1 is ubiquitously expressed and up-regulates the expressions of MMP-1, -3 , and -9 by interacting with multiple TLRs (23-26). In addition, HMGB1 functions as a nuclear DNA chaperone to support DNA replication within the nucleus (25, 27 ) and as a protein chaperone to prevent protein aggregation (28). HMGB1 can be translocated from the nucleus to the cytosol and then be extracellularly released during cell death, including necrosis, apoptosis, and pyroptosis (29). Several studies suggested that the synovial fluid level of HMGB1 is correlated with OA severity. Research using bovine cartilage and synovial fluid showed that the OARSI score of joints correlated with the extent of HMGB1 labeling in the ECM, chondrocyte nuclei, and cytoplasm, indicating that the switch of HMGB1 from a DNA-binding nuclear protein to an extracellular alarmin/cytokine correlates with the destruction of cartilage (30). In line with the suggested role of HMGB1 as a DAMP, HMGB1 neutralization by injection with an anti-HMGB1 antibody protected against lipopolysaccharideinduced lethality in mice (31). In our data, early treatment with 29-kDa FN-f increased the cellular level of HMGB1, and longer treatment significantly decreased the cellular level of HMGB1, because of a sharp increase in translocation of nuclear HMGB1 into the cytoplasm as well as extracellular release of HMGB1. Our result shows that 29-kDa FN-f may function as an amplifier of damage response in $\mathrm{OA}$ by up-regulating HMGB1 extracellular release.

We also found that $29-\mathrm{kDa} F N-f$ inhibits chondrocyte autophagy by inducing the formation of more of the $\mathrm{BCl}-2$ :Beclin-1 complex than of the HMGB1:Beclin-1 complex. Because chondrocytes maintain fine balance of synthesis and turnover of ECM, regulation of cell metabolism and survival by autophagy is considered an important mechanism for maintaining cartilage integrity (32). Decreased autophagy and cellularity with increased apoptosis were found in aged mouse knee joints (5). Human OA cartilage and animal OA models revealed highly expressed mTOR, increased apoptosis, and reduced expression of autophagic genes (33). Furthermore, mechanical injury also suppresses autophagy, with decrease of Beclin-1 and LC3 expression, in the superficial zone of cartilage, with induction of cell death and loss of soluble glycosaminoglycan (7). In contrast, another study showed that autophagy in the OA pathogenesis may function differently in young and OA cartilage, leading to chondrocyte protection or death depending on age (34).

Autophagy and apoptosis are modulated by both $\mathrm{BCl}-2$ and Beclin-1 expression levels and localization of the Bcl-2:Beclin-1 complex. In particular, $\mathrm{BCl}-2$ inhibits assembly of autophagogome structures by binding to Beclin-1, subsequently suppressing autophagy, whereas HMGB1 disrupts interaction of $\mathrm{BCl}-2$ :Beclin- 1 by competing with $\mathrm{BCl}-2$ for interaction with Beclin-1 and driving Beclin-1 to autophagosomes, leading to the autophagy pathway $(15,35)$. Several studies showed that the mTOR signaling pathway was a key regulator of autophagy in OA cartilage. Increased expression of mTOR in peripheral blood mononuclear cells of patients were related to disease activity represented by synovitis, and more significant up-regulation of mTOR levels was found in the lesional area of the OA cartilage than in the non-lesional area $(36,37)$. Beclin-1 overexpression mitigated the phsophoinositol-3 kinase (PI3K)/protein kinase B (AKT)/mTOR signaling pathway, which led to increased cell viability, and inhibition of apoptosis and MMP expression (38). Furthermore, inhibition of mTOR by treatment with rapamycin led to significant suppression of cartilage degeneration in a surgically induced OA mouse model and chondroprotection in human OA chondrocytes $(39,40)$. In our experiments, 29-kDa FN-f down-regulated LC3-II level by activating the mTOR signaling pathway. 
In summary, our results demonstrate that 29-kDa FN-f regulated the expression of HMGB1, the cytoplasmic translocation of HMGB1, and subsequent extracellular secretion of HMGB1. In addition, 29-kDa FN-f inhibits the autophagy pathway by modulating the mTOR/HMGB1-dependent pathway, thereby harming articular chondrocytes. Modulation of the autophagy pathway inhibited by 29-kDa FN-f may be a useful strategy in the treatment of OA cartilage degradation.

\section{MATERIALS AND METHODS}

Materials, quantitative real-time reverse-transcription polymerase chain reaction analysis, immunofluorescence microscopy, and IP assay are described in the Supplementary Materials.

\section{Primary chondrocytes isolation and culture}

OA cartilage samples were obtained from the knee joints of OA patients ( $n=20,74.1 \pm 5.4$ years of age) at the time of total knee replacement surgery. Patient diagnoses were based on the criteria set forth by the American College of Rheumatology. Normal cartilage samples were obtained from the femoral head of patients $(n=9,73.7 \pm 10.1$ years of age $)$ with femoral neck fractures and no known history of OA or RA. The collection and use of human tissue samples was reviewed and approved by the Institutional Review Board of Hallym University Sacred Heart Hospital, Anyang, Korea (approval number 2013-I022). All patients provided written informed consent for the use of the discarded cartilage samples.

Human primary chondrocytes were isolated from the articular cartilage and cultured as previously described (13).

\section{Western blot analysis}

Cell lysates were prepared with radioimmunoprecipitation assay (RIPA) lysis buffer, and western blot analysis was performed as described previously $(41,42)$.

\section{ELISA}

The level of HMGB1 released from chondrocytes into culture medium was measured using Human HMGB1 ELISA kits (Elabscience Biotechnology, Houston, TX, USA). Briefly, 100 $\mu \mathrm{l}$ of culture medium was added to each well of a 96-well plate, and $100 \mu$ lof biotinylated detection antibody was also added for $30 \mathrm{~min}$ at $37^{\circ} \mathrm{C}$. After washing with wash buffer, 100 $\mu \mathrm{l}$ of HRP conjugate was added to each well for $30 \mathrm{~min}$ at $37^{\circ} \mathrm{C}$ followed by incubation with substrate reagent for $15 \mathrm{~min}$ at $37^{\circ} \mathrm{C}$. The absorbance was measured at $450 \mathrm{~nm}$ using an ELISA reader.

\section{Statistical analysis}

Data are expressed as the mean \pm standard deviation (SD). Statistical analyses were performed using a Mann-Whitney $U$ test or two-way analysis of variance. A value of $\mathrm{P}<0.05$ was considered statistically significant.

\section{ACKNOWLEDGEMENTS}

This study was supported by a grant (HI16C0287) from the Korean Health Technology R\&D Project, Ministry of Health \& Welfare, Republic of Korea, the Basic Science Research Program through the National Research Foundation (NRF) of Korea funded by the Ministry of Education (2017R1 A2B2001881), and in part by the Hallym University Research Fund.

\section{CONFLICTS OF INTEREST}

The authors have no conflicting interests.

\section{REFERENCES}

1. Goldring MB and Goldring SR (2007) Osteoarthritis. J Cell Physiol 213, 626-634

2. Kim HA, Cho ML, Choi HY et al (2006) The catabolic pathway mediated by Toll-like receptors in human osteoarthritic chondrocytes. Arthritis Rheum 54, 2152-2163

3. Rahman MA and Rhim H (2017) Therapeutic implication of autophagy in neurodegenerative diseases. BMB Rep 50, 345-354

4. Loeser RF (2009) Aging and osteoarthritis: the role of chondrocyte senescence and aging changes in the cartilage matrix. Osteoarthritis Cartilage 17, 971-979

5. Carames B, Olmer M, Kiosses WB and Lotz MK (2015) The relationship of autophagy defects to cartilage damage during joint aging in a mouse model. Arthritis Rheumatol $67,1568-1576$

6. Carames B, Taniguchi N, Otsuki S, Blanco FJ and Lotz M (2010) Autophagy is a protective mechanism in normal cartilage, and its aging-related loss is linked with cell death and osteoarthritis. Arthritis Rheum 62, 791-801

7. Carames B, Taniguchi N, Seino D, Blanco FJ, D'Lima D and Lotz M (2012) Mechanical injury suppresses autophagy regulators and pharmacologic activation of autophagy results in chondroprotection. Arthritis Rheum 64, 1182-1192

8. Wang CC, Lee CH, Peng YJ, Salter DM and Lee HS (2015) Platelet-Rich Plasma Attenuates 30-kDa Fibronectin Fragment-Induced Chemokine and Matrix Metalloproteinase Expression by Meniscocytes and Articular Chondrocytes. Am J Sports Med 43, 2481-2489

9. Xie DL, Hui F, Meyers R and Homandberg GA (1994) Cartilage chondrolysis by fibronectin fragments is associated with release of several proteinases: stromelysin plays a major role in chondrolysis. Arch Biochem Biophys 311, 205-212

10. Xie DL, Meyers R and Homandberg GA (1992) Fibronectin fragments in osteoarthritic synovial fluid. J Rheumatol 19, 1448-1452

11. Homandberg GA and Hui F (1996) Association of proteoglycan degradation with catabolic cytokine and stromelysin release from cartilage cultured with fibronectin fragments. Arch Biochem Biophys 334, 325-331

12. Pichika R and Homandberg GA (2004) Fibronectin 
fragments elevate nitric oxide $(\mathrm{NO})$ and inducible $\mathrm{NO}$ synthetase (iNOS) levels in bovine cartilage and iNOS inhibitors block fibronectin fragment mediated damage and promote repair. Inflamm Res 53, 405-412

13. Hwang HS, Park SJ, Cheon EJ, Lee MH and Kim HA (2015) Fibronectin fragment-induced expression of matrix metalloproteinases is mediated by MyD88-dependent TLR-2 signaling pathway in human chondrocytes. Arthritis Res Ther 17, 320

14. Lee SA, Kwak MS, Kim S and Shin JS (2014) The role of high mobility group box 1 in innate immunity. Yonsei Med J 55, 1165-1176

15. Marquez RT and Xu L (2012) Bcl-2:Beclin 1 complex: multiple, mechanisms regulating autophagy/apoptosis toggle switch. Am J Cancer Res 2, 214-221

16. Davalos AR, Kawahara M, Malhotra GK et al (2013) p53-dependent release of Alarmin HMGB1 is a central mediator of senescent phenotypes. J Cell Biol 201, 613-629

17. Jeon $\mathrm{OH}, \mathrm{Kim} \mathrm{C}$, Laberge RM et al (2017) Local clearance of senescent cells attenuates the development of post-traumatic osteoarthritis and creates a pro-regenerative environment. Nat Med 23, 775-781

18. Ding L, Buckwalter JA and Martin JA (2017) DAMPs Synergize with Cytokines or Fibronectin Fragment on Inducing Chondrolysis but Lose Effect When Acting Alone. Mediators Inflamm 2017, 2642549

19. Yu C, Yu X, Zhu HW et al (2016) Expression pattern of HMGB1 and its association with autophagy in acute necrotizing pancreatitis. Mol Med Rep 14, 5507-5513

20. Rosenberg JH, Rai V, Dilisio MF, Sekundiak TD and Agrawal DK (2017) Increased expression of damageassociated molecular patterns (DAMPs) in osteoarthritis of human knee joint compared to hip joint. Mol Cell Biochem 436, 59-69

21. Yasuda T (2006) Cartilage destruction by matrix degradation products. Mod Rheumatol 16, 197-205

22. Homandberg GA, Wen C and Hui F (1998) Cartilage damaging activities of fibronectin fragments derived from cartilage and synovial fluid. Osteoarthritis Cartilage 6, 231-244

23. Fang $F$ and Jiang D (2016) IL-1beta/HMGB1 signalling promotes the inflammatory cytokines release via TLR signalling in human intervertebral disc cells. Biosci Rep 36,

24. Park JS, Gamboni-Robertson F, He Q et al (2006) High mobility group box 1 protein interacts with multiple Toll-like receptors. Am J Physiol Cell Physiol 290, C917-924

25. Stros M (2010) HMGB proteins: interactions with DNA and chromatin. Biochim Biophys Acta 1799, 101-113

26. Taniguchi N, Kawakami Y, Maruyama I and Lotz M (2018) HMGB proteins and arthritis. Hum Cell 31, 1-9

27. Javaherian K, Liu JF and Wang JC (1978) Nonhistone proteins HMG1 and HMG2 change the DNA helical structure. Science 199, 1345-1346

28. Min HJ, Ko EA, Wu J et al (2013) Chaperone-like activity of high-mobility group box 1 protein and its role in reducing the formation of polyglutamine aggregates. J Immunol 190, 1797-1806

29. Magna M and Pisetsky DS (2015) The Role of Cell Death in the Pathogenesis of SLE: Is Pyroptosis the Missing Link? Scand J Immunol 82, 218-224

30. Heinola T, de Grauw JC, Virkki L et al (2013) Bovine chronic osteoarthritis causes minimal change in synovial fluid. J Comp Pathol 148, 335-344

31. Wang $\mathrm{H}$, Bloom O, Zhang M et al (1999) HMG-1 as a late mediator of endotoxin lethality in mice. Science 285, 248-251

32. Almonte-Becerril $M$, Navarro-Garcia $F$, Gonzalez-Robles A, Vega-Lopez MA, Lavalle C and Kouri JB (2010) Cell death of chondrocytes is a combination between apoptosis and autophagy during the pathogenesis of Osteoarthritis within an experimental model. Apoptosis 15, 631-638

33. Zhang Y, Vasheghani F, Li YH et al (2015) Cartilagespecific deletion of mTOR upregulates autophagy and protects mice from osteoarthritis. Ann Rheum Dis 74, $1432-1440$

34. Chang J, Wang W, Zhang $\mathrm{H}, \mathrm{Hu} Y$, Wang $M$ and Yin Z (2013) The dual role of autophagy in chondrocyte responses in the pathogenesis of articular cartilage degeneration in osteoarthritis. Int J Mol Med 32, 1311-1318

35. Tang D, Kang R, Livesey KM et al (2010) Endogenous HMGB1 regulates autophagy. J Cell Biol 190, 881-892

36. Katsara O, Attur M, Ruoff R, Abramson SB and Kolupaeva V (2017) Increased Activity of the Chondrocyte Translational Apparatus Accompanies Osteoarthritic Changes in Human and Rodent Knee Cartilage. Arthritis Rheumatol 69, 586-597

37. Tchetina EV, Poole AR, Zaitseva EM et al (2013) Differences in Mammalian target of rapamycin gene expression in the peripheral blood and articular cartilages of osteoarthritic patients and disease activity. Arthritis 2013, 461486

38. Song B, Song $\mathrm{H}$, Wang $W$ et al (2017) Beclin 1 overexpression inhibits chondrocyte apoptosis and downregulates extracellular matrix metabolism in osteoarthritis. Mol Med Rep 16, 3958-3964

39. Carames B, Hasegawa A, Taniguchi N, Miyaki S, Blanco FJ and Lotz M (2012) Autophagy activation by rapamycin reduces severity of experimental osteoarthritis. Ann Rheum Dis 71, 575-581

40. Zhang T, Liu J, Zheng X, Zhang B and Xia C (2018) Different roles of Akt and mechanistic target of rapamycin in serumdependent chondroprotection of human osteoarthritic chondrocytes. Int J Mol Med 41, 977-984

41. Hwang HS, Park IY, Kim DW, Choi SY, Jung YO and Kim HA (2015) PEP-1-FK506BP12 inhibits matrix metalloproteinase expression in human articular chondrocytes and in a mouse carrageenan-induced arthritis model. BMB Rep 48, 407-412

42. Yusein-Myashkova S, Ugrinova I and Pasheva E (2016) Non-histone protein HMGB1 inhibits the repair of damaged DNA by cisplatin in NIH-3T3 murine fibroblasts. BMB Rep 49, 99-104 